\title{
Ejercicio físico, salud y supuestos en el cálculo de la frecuencia cardíaca máxima estimada
}

\section{Exercise, Health and Assumptions in Calculating the Estimated Maximum Heart Rate}

\author{
Alixon David Reyes Rodríguez. \\ Universidad Pedagógica Experimental Libertador \\ Instituto Pedagógico de Maturín \\ Maturín, Venezuela \\ alixdavid79@yahoo.com
}

Recibido 11 de noviembre de 2010 • Aceptado 09 de marzo de 2011

\begin{abstract}
Resumen. En tiempos en los que se habla ya de nanociencia, de nanotecnología, de biología sistémica, de reingeniería genética, de ecoalfabetización, de ecoeconomía, de revolución biotecnológica, etc., pareciera que aún permanecen viejas escuelas con supuestos científicos y referentes teóricos que en su momento dieron respuestas a necesidades científicas, pero que ya hoy son insuficientes. Se menciona esto porque resulta un atraso para la ciencias de la educación y para las ciencias del deporte el hecho de que aún se sigan enseñando en congresos nacionales e internacionales, seminarios, jornadas de investigación, en nuestras universidades y en cualquier tipo de reuniones científicas, algunos supuestos que han perdido vigencia y que, por lo tanto, han caducado en cuanto a su legitimidad y validez. Reconocemos los avances que para la ciencia del ejercicio físico y la fisiología del ejercicio representaron, en su momento, algunas fórmulas predictivas para el cálculo de la frecuencia cardíaca máxima estimada (FCME), hay aspectos dignos de considerar que atienden a la necesidad. No que las despreciemos, lo que se pretende a través del presente trabajo es mostrar y desmitificar el hecho de la fórmula tradicional como casi el único patrón de medición y cálculo de la FCME, y más allá ofrecer, desde la perspectiva de otros investigadores, posibilidades para una mejora en cuanto a la dosificación del ejercicio físico para ciertos tipos de poblaciones con especificidades singulares.
\end{abstract}

Palabras claves. Frecuencia cardíaca máxima estimada, ejercicio físico, dosificación, predicción, Karvonen.

\footnotetext{
Profesor de Educación Física, Deporte y Recreación, Magíster en Enseñanza de la Educación Física, actualmente candidato a Doctor en Educación y Maestrante en Educación Superior. Profesor de la Universidad Pedagógica Experimental Libertador (República Bolivariana de Venezuela) en la asignatura de Gimnasia artística masculina. Ha trabajado con otras asignaturas como Fundamentos teóricos de la Educación Física, Principios científicos del entrenamiento, Educación Física y salud. Profesor en el postgrado de la universidad, tutor, jurado y asesor. Adelanta proyectos en al campo de la Antropología del cuerpo, motricidad y lúdica, entre otros. Asimismo, se desempeña como Coordinador General del Núcleo de Investigación en Pedagogía del Movimiento y como Director-Editor de la Revista Científica EDUCACIÓN EN MOVIMIENTO. En la actualidad es miembro de la Confederación Iberoamericana de Medicina del Deporte.
} 
Abstract. Today, when the discussions are about Nanoscience, Nanotechnology, Systems Biology, Genetic Engineering, Ecological Literacy, Ecological Economics, Biotechnological Revolution, among others, it seems that there are still old schools with scientific assumptions and theoretical points of reference that responded to scientific needs before, but which are insufficient now. It has been observed in national and international conferences, seminaries, research encounters, in our universities and in different kinds of scientific meetings that some obsolete assumptions are still being taught, which slows down progress in Education Sciences and Sports Science. We recognize that some predictive formulas used to calculate the estimated maximum heart rate (EMHR) represented progress for Exercise Science and Exercise Physiology, at some point; however, there are important aspects that should be considered. It is not that we despise them, but we intend to demonstrate and demystify the use of the traditional formula almost as the only calculation and measurement pattern for EMHR and, to offer, from the perspective of other researchers, better possibilities of exercise dosage for certain populations with particular characteristics.

Keywords. Estimated Maximum Heart Rate, exercise, measurement, prediction, Karvonen.

\section{Introducción}

El presente trabajo ofrece una revisión de bibliografía especializada tomando como referencias algunas investigaciones que en el campo de la fisiología del ejercicio permiten desmitificar supuestos científicos relacionados con la determinación de la frecuencia cardíaca máxima estimada (FCME) como parámetro indicador para la dosificación de la intensidad en el delicado trabajo de la prescripción del ejercicio físico a través de ecuaciones predictivas para su cálculo en variadas poblaciones. Se parte de la FCME, habida cuenta de su carácter fundamental como patrón de medición.

Como punto de partida se considera que ir a la par de los avances científicos ofrece al ser humano la posibilidad de mejorar los procesos que conduce. En el campo de las ciencias aplicadas al deporte se ha visto, con el pasar de los años, una evolución tecnológica que permite disminuir los umbrales del rendimiento humano, a la vez que se disminuyen los riesgos externos de la actividad física. Hoy se cuenta con analizadores de gases, con tapices rodantes, con túneles de viento, con cámaras hiperbáricas, plataformas de fuerza, pulsómetros, ergómetros, cicloergómetros, remoergómetros, sistemas de video-análisis, sistemas de grabación, fotocélulas, controles genéticos, GPS para monitorear el esfuerzo y el rendimiento físico en el hecho deportivo, trajes hechos con materiales ergonómicos, etc. Sin duda alguna, estas aplicaciones tecnológicas han revolucionado el entrenamiento deportivo y, por supuesto, han contribuido a la optimización de las marcas y los registros de los atletas en competencias oficiales al potenciar su rendimiento de forma exponencial. Por supuesto, también han modificado las formas clásicas de entender y trabajar con la dosificación del ejercicio físico en el deporte de alto rendimiento.

No obstante, y a pesar de que se evidencian altos cotos de beneficios desde el contexto de la tecnología deportiva y el consiguiente avance de las ciencias aplicadas al deporte, estos no se democratizan y no son aprovechables en la magnitud esperada en otro tipo de poblaciones, sean estas poblaciones con problemas cardiovasculares o no. Incluso, en materia de ciencias aplicadas al deporte existen ciertos avances para los cuales (a efectos de su aprovechamiento) ya no se precisan máquinas o aparatos, laboratorios o espacios amplios y acondicionados de forma específica. En este trabajo se toman en cuenta algunos avances evidenciados en lo que respecta al cálculo de la FCME a través de las ecuaciones o fórmulas predictivas.

Cuando se habla de la democratización de la Ciencia, se destaca la necesidad del aprovechamiento popular de los hallazgos resultantes de las investigaciones, es decir, ello debe 
impactar en la sociedad civil, sin quedarse en los espacios recónditos de las comunidades científicas. Lo contrario no tiene sentido la investigación. Si no hay correspondencia con la población en general o, en todo caso, con la población afectada, la investigación pierde sentido. Al respecto, se hace necesario el uso y empleo del conocimiento derivado de la Ciencia de forma global, puesto a la orden de las escuelas, de las universidades, de las unidades básicas de investigación; a la orden de las federaciones deportivas, de las asociaciones, de las ligas y de los clubes, de los entrenadores y del resto del personal técnico, de los atletas, de los profesores y estudiantes de áreas tan álgidas en el contexto del deporte como son: la Educación Física, la Recreación y las Ciencias Aplicadas al Deporte. ¿De dónde surge ese tipo de conocimientos específicos?, pues, de las universidades, de los centros nacionales e internacionales de ciencias aplicadas al deporte, de laboratorios destinados a la investigación científica con aplicaciones a la tecnología deportiva.

\section{Actividad física, salud y políticas públicas}

Aunque ya parece ser un slogan publicitario, vale recordar que, desde hace algún tiempo, la actividad física estructurada ha sido reconocida como una fuente de vida y salud por grandes organismos: la Organización de las Naciones Unidas para la Educación, la Ciencia y la Cultura (UNESCO) y la Organización Mundial de la Salud (OMS). Consecuentemente, otras organizaciones: la Federación Mundial del Corazón (WHF -siglas en inglés-), la Sociedad Latinoamericana de Cardiología Intervencionista (SOLACI), la Federación Internacional de la Educación Física (FIEP), la Federación Internacional de Actividad Física Adaptada (IFAPA), el Colegio Americano de Medicina del Deporte (ACSM -siglas en inglés-), la Federación Internacional de Medicina del Deporte (FIMS), el Consejo Internacional de Ciencias del Deporte y Educación Física (ICCSPE), la Asociación Americana de Cardiología (AHA -siglas en inglés-), entre otros, también están de acuerdo y han aconsejado, a la población en general, a través de investigaciones, comunicados y manifiestos, congresos científicos y seminarios, en los que estimulan la realización de actividad física de forma sistemática y periódica en tiempos en los que "la inactividad física sigue siendo un importantísimo problema de salud" (Abellán, Sainz, Ortín, 2010, p. 11).

No obstante estas consideraciones, la OPS (2007) informó que: "las Enfermedades Cardiovasculares son la primera causa de muerte a nivel mundial: más personas mueren anualmente de las Enfermedades Cardiovasculares que de otra causa (...)" (I 18), y estima que "para el 2015, casi 20 millones de personas morirán de las Enfermedades Cardiovasculares” (I18). Colin Mathers, Coordinador de Epidemiología y Carga de Morbilidad de la OMS (2008) y autor de un estudio al respecto, ya anunció que para el año 2030 las enfermedades cardiovasculares se convertirían en la primera causa de muerte en América Latina y a nivel mundial. No tuvo que pasar mucho tiempo para que la Organización Panamericana de la Salud (OPS) revelase datos sumamente alarmantes. Finalmente, en 2009, la American Heart Association [AHA] (s. f.) constató las afirmaciones de la OPS (2007), esto es, que las enfermedades cardiovasculares se habían convertido -para este tiempo- en la primera causa de muerte a nivel mundial.

No es un secreto de estado el hecho que en muchos países -que no todos- existe una tendencia al alza en las tasas de enfermedades cardiovasculares -ya ha sido pronosticado por importantes organizaciones (OMS, 2008)-, pero aunado a ello se tiene que la implosión ocasionada por estilos de vida cada vez más sedentarios, el secuestro de las áreas verdes y los parques naturales por parte de los edificios, las calles de asfalto y cemento endurecido, el exceso de trabajo, lo atestado de las vías y el tránsito, la inseguridad en las calles, la contaminación, el manejo inadecuado, el aumento 
exacerbado de ejemplares vehiculares y la dependencia excesiva a las tecnologías de información y comunicación; todo ello ha llenado de preocupación a las autoridades sanitarias y educativas de diversas naciones. En tal sentido, los gobiernos nacionales deben diseñar políticas de atención social destinadas a la disminución y control de ciertos factores de riesgo como el sedentarismo, la obesidad, el uso del tabaco, la diabetes, hábitos alimenticios inadecuados, la hipercolesterolemia, entre otros. De todos estos factores de riesgo, uno llama la atención de forma preponderante: el sedentarismo.

En países como Brasil se han desarrollado políticas públicas que tienen programas destinados a disminuir las tasas de sedentarismo existentes, programas que como Agita Sao Paulo son implementados involucrando a la mayor cantidad posible de personas. Este programa impulsado en Sao Paulo (Brasil), por el Dr. Victor Matsudo y su esposa la Dra. Sandra Matsudo, proponía a la población la realización de cualquier tipo de actividad física saludable por un período de 30 minutos diarios: caminar, correr, nadar, subir y bajar escaleras, saltar la cuerda, lavar los platos, ir al supermercado caminando, montar bicicleta, bañar el perro, entre otras. Además de estos, lanzaron folletos instructivos en alusión a los beneficios de la actividad física, concursos, competencias de conocimientos en alusión a la nutrición y el ejercicio físico.

Existen otros países con programas interesantes en la promoción de políticas públicas relacionadas con la salud a partir de la realización de ejercicio físico. Dinamarca es el país con la mayor proporción -a nivel mundial- de uso de la bicicleta en contraste con el uso del automóvil; en la ciudad de Bogotá (Colombia) se ha desarrollado una interesante red de ciclovías, en Venezuela se mantiene la Misión Barrio Adentro Deportivo, etc. No obstante, aún así parece ser poco para lograr contener la amenaza futura. Pareciera que tanto la población como los profesionales del área de la salud aún no se apropian definitivamente de la importancia de la adopción de estilos de vida físicamente activos, y atención: esto pasa por comprender la importancia y pertinencia de hacer actividad física de manera periódica y planificada, y pasa por decidir modificar los hábitos de vida que a diario obstaculizan la separación de tiempo para dedicarlo a la actividad física.

\section{Democratización de la Ciencia versus supuestos científicos}

Ahora bien, se presenta una disyuntiva para los profesionales de la salud que conscientemente trabajan para promover estilos de vida físicamente activos. Es curioso observar cómo aún se mantienen vigentes supuestos que en el campo de las ciencias aplicadas al deporte ya muestran signos de fractura y resquebrajamiento. Algunas personas, intentando adoptar estilos de vida físicamente activos, tratan de realizar cualquier tipo de actividad física y buscan información; sin embargo, en muchos casos encuentran a otras personas que haciendo el papel de instructores, entrenadores personales, incluso profesores, quienes lamentablemente desconocen mucho de las teorías generales del acondicionamiento físico y de la fisiología del ejercicio. Esto genera un grave problema, por cuanto la persona que decide hacer actividad física confía en quien se la administra y dosifica.

Muchas personas -que no todas, y tampoco la mayoría- que se dedican al trabajo de la prescripción y dosificación de actividad física no poseen los conocimientos básicos para dedicarse a esto. Esto podría tener varias causas: no haber sido formadas en el área, trabajar desde la base de la empiria (lo más frecuente) sin mayor sustento teórico-científico, falta de interés por documentarse en el tema, desconocimiento del manejo de tecnologías de información y comunicación, entre otros factores. Pero más preocupante aún es cuando esto sucede en el espacio académico, es decir, cuando en las universidades e instituciones de formación docente y profesional en el área de la salud y 
la actividad física, a los jóvenes estudiantes se les sigue enseñando y suministrando información errónea que fortalece una tradicional carencia formativa (Abellán et al. 2010). Y es que tal limitación formativa se agranda debido a varios aspectos, sin embargo, el que consideramos más importante tiene que ver con el poco ejercicio de la investigación por parte de profesores universitarios formados y formadores en el campo de la actividad física en América Latina (y para ser más específicos, en la República Bolivariana de Venezuela). Se está claro en que, aunque la actualización de contenidos no es el propósito principal de la investigación científica, no puede negarse su importancia y pertinencia en el contexto universitario. El creciente número de investigaciones, la creciente demanda de publicaciones científicas y el posterior surgimiento de las mismas -como consecuencia de lo anterior- ofrece una ventana para -por lo menos- actualizar contenidos. Entonces, a juicio de quien escribe estas líneas, no se justifica la permanente desactualización de contenidos en los programas de ciertos cursos y, peor aún, la de varios profesores. En este sentido, Lagardera y Lavega (2003) sostienen que "la ignorancia sigue siendo hoy tan osada y desvergonzada como antaño, de ahí que se cuele sin remilgos hasta en instituciones tan prestigiosas como la universidad" (p. 13). Es precisamente acá cuando se habla de estos supuestos en el entramado de la salud, el ejercicio físico y las fórmulas predictivas para el cálculo de la FCME.

Los avances científicos nos han demostrado la capacidad de investigación de la humanidad y sería hasta abusivo caer en terreno harto conocido al enumerar los beneficios que la ciencia aplicada ha deparado al deporte. No obstante, se hace necesario democratizar el conocimiento científico de manera tal que la población destinataria pueda hacerse de los conocimientos necesarios. En esta ocasión y para este trabajo, tan solo se ha de destacar el supuesto de la fiabilidad en las ecuaciones o fórmulas predictivas para el cálculo de la FCME, aplicado a cualquier población.

\section{Supuestos en las fórmulas predictivas para el cálculo de la FCME}

La FCME viene a representar un parámetro indicador para la determinación y la dosificación del ejercicio físico en cuanto a sus variables dinámicas -frecuencia, intensidad, volumen- (Subiela, 2005). Así mismo, pasa a ser una constante-variable (por efectos de la individualidad) del trabajo cardiovascular que bajo ninguna excusa debe soslayarse.

Para determinar la FCME se han utilizado, históricamente, las fórmulas y ecuaciones que permiten predecir el estatus del trabajo cardíaco en el contexto de la actividad física deportiva. Sin embargo, algunas tendencias que distan de no ser polémicas levantan no pocas sospechas y la preocupación en el mundo científico especializado con respecto al empleo de estas ecuaciones.

Existe una ecuación que ha despertado mucha discusión y que de paso ha visto disminuido el uso que se le ha dado en el grupo de fisiólogos y otros científicos que trabajan como auxiliares de las ciencias deportivas. Esta ecuación ha ido perdiendo vigencia, porque ya su uso no es tan extendido en el grupo de profesionales que trabajan con la dosificación del entrenamiento deportivo en atletas de alto rendimiento a nivel internacional. No obstante, la preocupación radica en que la ecuación o fórmula predictiva para el cálculo de la FCME según la cual esta es igual a 220 - edad, sigue siendo enseñada en varias universidades e institutos formadores de profesionales que atenderán a poblaciones a las cuales tendrán que prescribirles actividad física de forma sistemática, ya sea en el entorno escolar o en otro entorno. Ahora bien, este estudio considera que esa fórmula no es práctica en estos tiempos, por la sencilla razón de que no respeta ni corresponde a los principios básicos del entrenamiento físico - principios que han sido pensados desde la base de investigaciones contrastadas con el paso de la historia-. Si se juzga que a razón de tal fórmula se puede dosificar el 
ejercicio físico de forma adecuada, se podría cometer un severo error al no respetar el principio de individualidad. A continuación, un primer ejemplo que quizás podría parecer exagerado:

Se tienen dos sujetos: A y B. Ambos tienen 22 años de edad. El sujeto A forma parte de la Selección Nacional de Atletismo y trabaja con un plan de entrenamiento ejecutado dentro del ciclo olímpico de cara a los próximos Juegos Olímpicos de Londres 2012. Al intentar calcular su FCME se le aplicaría la fórmula anteriormente señalada y daría los siguientes resultados:

Sujeto A

$$
\begin{gathered}
220-\text { Edad }=\text { FCME } \\
220-22=198
\end{gathered}
$$

Siendo así, este sujeto, que presenta una FCME de 198 pulsaciones por minuto, tendría que planificar el entrenamiento deportivo pensando en estratificar la carga por niveles porcentuales. Si desea trabajar a un $60 \%$, deberá multiplicar 198 por 0,6 , lo cual da un resultado de 118,8, que con una aproximación al superior inmediato llega a ser de 119 pulsaciones por minuto.

No obstante, retornando a la idea original, se tiene que, de acuerdo con esta fórmula, la FCME del sujeto A -joven atleta miembro de la Selección Nacional de Atletismo- está ubicada en 198 pulsaciones por minuto.

Ahora, los datos del Sujeto B. Este último tiene igualmente 22 años de edad, no obstante, tiene 5 años postrado en cama debido a un accidente automovilístico que le dejó parapléjico. Al aplicarle la fórmula tendremos que los resultados serán idénticos a los de sujeto A. En este sentido se puede preguntar: ¿Cómo puede entonces ser eso posible?

El sujeto A está siendo sometido a una carga de entrenamiento continua de alta exigencia, mientras que el sujeto B tiene severos problemas de salud y padece una inmovilidad forzada desde hace cinco años. Es bien sabido que el ejercicio físico periódico produce alteraciones y adaptaciones anatómico-fisiológicas importantes en el organismo de quien se ejercita. Estas alteraciones, al ser anatómicas, se traducen en aspectos cuantitativos; mientras que las alteraciones de carácter fisiológico se traducen en aspectos cualitativos importantes (a menos que la persona en cuestión use betabloqueadores - por cuanto la frecuencia cardíaca no aumenta a causa de los estímulos del ejercicio físico - o tenga instalado un marcapasos - debido a que la frecuencia cardíaca está configurada y controlada de manera intencional-). Por ejemplo, cuando por efectos del ejercicio físico periódico y sistemático se disminuye la frecuencia cardíaca en estado de reposo basal, se está en presencia de un mayor flujo sanguíneo, a la vez que se evidencia un menor trabajo cardíaco, por cuanto las contracciones miocárdicas se prolongan más (Subiela, 2005).

Esas adaptaciones anatómico-fisiológicas no podrán ser alcanzadas por el sujeto B, mientras que, por el contrario, el sujeto A tiene la mayor factibilidad de lograrlo. De partida esta situación hace que el sujeto A mejore sus posibilidades cardiovasculares y respiratorias de una forma que no podrá ser igual a la del sujeto B. Entendiendo esto es fácil establecer que el umbral de la FCME en ambos sujetos será diferente y favorecerá, primordialmente, al sujeto A.

Este primer ejemplo permite verificar, de forma sencilla y práctica, algunos aspectos puntuales. En primer lugar, el estado de salud, el nivel de condición física y la toma en cuenta de la frecuencia cardíaca estimada (FCE) en estado de reposo basal, son factores preponderantes que destruyen la idea de amalgamar la fórmula predictiva $(220$-edad = FCME). En segundo término, la constante de 220 no es funcional, porque traza un umbral precisamente igual para personas de diferentes edades (lo único que lo diferencia es la edad) y eso no resulta ser siempre así. Puede darse 
el caso de personas físicamente activas con edades entre 50 y 60 años que registren una FCE en estado de reposo relativo con valores inferiores a personas con edades de 40 años (y menos) que son sedentarios crónicos. Como podrá verse, entonces, el umbral fisiológico responderá a otros factores que no se circunscriben a la edad de las personas, por ejemplo: el tipo de actividad, la frecuencia de la actividad, la intensidad de trabajo, el protocolo de evaluación, entre otros.

Ahora bien, si se compara a dos personas de la misma edad que practiquen una misma disciplina deportiva se podría conseguir otros aspectos importantes. Por ejemplo, el fútbol. Se piensa en dos jugadores de la misma edad que juegan en posiciones diferentes: en tal caso se tiene como referencias a un portero y a un lateral de características y vocación ofensiva. A pesar de que en las sesiones de entrenamiento ambos jugadores tengan tareas prácticas de carácter aeróbico, no hace falta mucha elucubración para entender que en un juego de fútbol oficial el lateral va a hacer un recorrido mayor al recorrido del portero en cuestión. Además, cada uno de estos jugadores tendrá que hacer trabajos diferenciados en el entrenamiento, debido a las características y a las demandas de la posición en la que juega.

Es preciso comprender que en un juego oficial el jugador lateral hace un trabajo constante de 90 minutos con características mixtas (aeróbico-anaeróbico) y logra altas distancias de recorrido al final del juego; mientras que un portero -debido a las características y exigencias de su posición- no realiza la misma magnitud con respecto al desplazamiento. A la recurrente pregunta: ¿se ven ambos jugadores a forzar el umbral de la FCME?, la respuesta es no. Es claro que el jugador lateral sí es empujado a forzar el umbral de la FCME, debido a las demandas del juego; mientras que el portero no es forzado en la misma proporción. Al forzar el umbral de la FCME es mucho más factible que el jugador lateral desarrolle mejores condiciones para el desarrollo aeróbico, en contraste con el portero, y eso hace una gran diferencia al momento de calcular la FCME.

En una investigación realizada sobre la distancia recorrida por los futbolistas en un juego, Valenzuela (2007) sostiene que:

En total un jugador de fútbol a lo largo de los 90 minutos recorre entre 9 y 13 o 14 kilómetros como volumen total, abarcando todas las acciones, caminar, trotar, picar, etc. Los centrales y los puntas son en general los que menos recorrido realizan, entre 9.300 y 10.000 metros. Los medios y carrileros los que más recorren, cerca de 13.000 metros los primeros y 12.000 los segundos. (J5)

Cuando Valenzuela (2007) menciona a "los carrileros" se refiere a los laterales. Y como podrá verse, estos se encuentran entre los que mayor recorrido realizan en un juego de fútbol. A los que Valenzuela llama medios son los jugadores mediocampistas. Justo después de transcurridas las dos primeras jornadas en el Mundial de Fútbol de Sudáfrica 2010, las estadísticas que ofrecía la FIFA (citadas por Valenzuela 2007) mostraban que los únicos jugadores que sobrepasaban los 11,5 kilómetros recorridos como promedio de los dos primeros juegos eran todos mediocampistas, a saber: el australiano Carl Valeri con 24,82 kilómetros (Kms), el mexicano Gerardo Torrado con 24,22 Kms, el alemán Sami Khedira con 24,16 Kms., el estadounidense Michael Bradley con 23,82 Kms, y el alemán Bastian Schweinsteiger con 23,60 Kms.

Ahora bien, los porteros son los que menos distancia recorren en un juego, por cuanto deben defender el arco y ello amerita mantener la posición durante todo el tiempo de duración del partido (salvo contadas excepciones en las que deben salir del área a cortar una jugada que conlleva peligro al arco). Esto lleva a comprender que el gasto energético de un lateral y el gasto energético de un portero no serán similares, por tanto, la FCE y la FCME han de diferenciarse entre ambos deportistas, a pesar de que practiquen una misma disciplina deportiva. 
En el caso -ahora- de un velocista (corredor de 100 metros planos) y el caso de un receptor en el béisbol, ambos con la misma edad, a simple vista se nota una gran diferencia entre ambos deportistas debido al nivel de exigencia física -que no exigencias técnicas- de cada una de las disciplinas. Mientras que el velocista es forzado debido a las características de su disciplina y de la prueba en particular a trabajar con capacidades máximas y sub-máximas, tanto en entrenamientos como en competencias oficiales, un receptor en el béisbol no se verá forzado a desarrollar trabajos físicos de la misma magnitud. En tal sentido, lo que se desea mostrar es que el cálculo de la FCME no puede aplicarse con los mismos parámetros a este tipo de poblaciones, aun siendo todos deportistas, y que una misma ecuación (fórmula) predictiva para el cálculo de la FCME no es aplicable tampoco a distintas poblaciones; tampoco se puede garantizar que sea aplicable a deportistas que practiquen la misma disciplina, porque en todo caso las exigencias de deportes de posición como el fútbol, el béisbol, entre otros, ha de variar el umbral de la FCME en los mismos deportistas.

Hasta acá parece estar claro que el cálculo de la FCME no puede pensarse ya a estas alturas del siglo XXI desde la base de esa fórmula originaria (220 - Edad), en el caso tácito de que:

$\checkmark$ Se comprueba su falta de fiabilidad.

$\checkmark$ La sola aplicación de la ecuación en personas de la misma edad -aunque en condiciones diferentes- muestra signos de falsabilidad o falsacionismo (Popper, 1985).

$\checkmark$ La aplicación de la ecuación en una misma población hace ver que los resultados no son referenciales.

$\checkmark$ Existen ciertos factores que influyen poderosamente en la variabilidad de la FCME, tales como la edad, el estilo de vida de la persona en cuestión, el tipo de actividad a la que se dedica mientras hace actividad física, el tiempo de dedicación, la sistematicidad de la actividad física que se realiza, la obesidad, el protocolo de evaluación, etc.

A todas estas, se puede afirmar que aplicar esta ecuación generalizando las poblaciones no es lo más conveniente. Al respecto, se hace necesario revisar la experiencia de otros autores e investigadores que en el campo de la fisiología del ejercicio han mostrado avances y han hecho influyentes aportes en el campo de esta ciencia de las denominadas aplicadas al deporte.

El Dr. Martii Karvonen propuso la ecuación que permite calcular la frecuencia cardíaca de reserva y conmocionó al mundo de la fisiología y por supuesto de las ciencias aplicadas al deporte. Originó una gran revolución a finales de la década de los 50. Esta fórmula ha permitido reconocer uno de los principios científicos del entrenamiento físico considerados como fundamentales: la individualidad. Tal y como lo describen Aracil y Moya (2010):

En el ámbito de la actividad física y del deporte, la figura de Karvonen está comúnmente ligada al desarrollo de la fisiología del ejercicio en los momentos en los que esta disciplina vivía su consolidación como tal, a mediados del siglo XX. Su contribución más destacada en este campo fue el desarrollo del concepto de "frecuencia cardiaca de reserva", publicado en 1957 (Karvonen, Kentala y Mustala, 1957), que consiste en la diferencia entre la frecuencia cardiaca basal y la frecuencia cardiaca máxima de un individuo. A partir de este concepto, se derivó la fórmula de estimación de la intensidad del esfuerzo cardiovascular, popularmente conocida como la "fórmula de Karvonen". (p. 81) 
La fórmula de Karvonen sugiere el cálculo de la frecuencia cardíaca de reserva (FCR), la cual se obtiene de la resultante entre la resta de la FCR y la frecuencia cardíaca en reposo (FCRp). Se representa con la siguiente ecuación: FCR = FCM - FCRp. Es de destacar que los resultados de las fórmulas predictivas ofrecidas se corresponden con los valores del cálculo del cociente respiratorio y del cálculo del consumo máximo de oxígeno $\left(\mathrm{VO}_{2}\right)$ en trabajos personalizados. Incluso, hasta para el cálculo de otros factores como el ritmo cardíaco, la reserva de ritmo cardíaco y el ritmo cardíaco de entrenamiento, las fórmulas predictivas de Karvonen mantienen vigencia, a saber:

\begin{tabular}{l}
\hline \multicolumn{1}{c}{ Ritmo cardiaco máximo teórico $(\mathrm{RCMT})$} \\
RCMT Mujeres $=214-(0.8 \times$ Edad $)$ \\
RCMT Hombres = $209-(0.7 \times$ Edad $)$ \\
\hline Reserva de ritmo cardiaco $($ RRC $)$ \\
RRC = RCMT - Ritmo cardiaco basal \\
Ritmo cardiaco de entrenamiento $($ RCE $)$ \\
RCE $=($ RRC $\times$ Intensidad $)+$ Ritmo cardiaco basal \\
\hline
\end{tabular}

Aparte de ello y para confirmar lo anterior, Mujika (2006) sostiene esta idea al asegurar que "la frecuencia cardiaca muestra una respuesta al ejercicio similar a la del consumo de oxígeno, por lo que puede utilizarse de manera parecida para estimar la intensidad del esfuerzo (...)" (p. 5). No obstante, aún muchas personas insisten en seguir generalizando patrones de predicción como si los resultados realmente fuesen viables. Precisamente y con respecto a esto, Garatachea, García y de $\mathrm{Paz}$ (2005) sostienen que en efecto "la relación entre la $\mathrm{FC}$ y el $\mathrm{VO}_{2}$ varía entre sujetos, y existe acuerdo en que se determine de forma individual" (p. 132). En un estudio realizado por Bouzas, Ottoline y Delgado (2010), referente a las aplicaciones de la FCME en la evaluación y prescripción de ejercicio físico, los autores llegan a la siguiente conclusión:

La estimación del $\mathrm{VO}_{2 \max }$ por medio de la FCM predicha por una ecuación inadecuada puede producir importantes errores a la hora de prescribir un entrenamiento: cargas de entrenamiento sin efectividad para proporcionar adaptaciones orgánicas o cargas extremadamente elevadas que pueden contribuir a desarrollar un estado de sobreentrenamiento (...). (p. 252)

En estudios realizados por Silva-Junior, Xavier y Bouzas en la especialidad de ciclismo (2003); Nogueira, Xavier, Figuereido y Bouzas, en un estudio en la especialidad de natación en estilo libre (2003); Freitas, Xavier, Silva-Junior y Bouzas en la especialidad de carrera (2003), y Bouzas y Delgado (2004) en un estudio sobre perfiles aeróbicos y anaeróbicos, se hacen comparaciones de la FCME calculada a través de 21 ecuaciones diferentes. Bouzas (2008), en un trabajo posterior (tomando en cuenta que él formó parte de tales estudios), manifiesta que los investigadores de aquellas investigaciones comprobaron que "existen ecuaciones específicas para cada actividad" (p. 94) y, además de ello, comprobaron "que la mayoría de las ecuaciones no estimaban adecuadamente la FCM por los deportistas" (Bouzas, 2008, p. 94). Esos resultados permiten desmitificar el uso de la fórmula tradicional para el cálculo de la FCME (220 - Edad) para poblaciones globales, y de por sí ya representa un adelanto sustancial en el contexto de la dosificación del ejercicio físico. Se considera que las ciencias aplicadas al deporte han avanzado mucho con respecto a los aportes de la fisiología del ejercicio. Y, precisamente, desde ese campo se reciben alertas sobre la imposibilidad de generalizar el cálculo de la FCME, porque de lo contrario se podría incurrir en equivocaciones 
metodológicas al momento de prescribir ejercicio físico. Las peores consecuencias las padecerán aquellos a quienes se les administre la actividad física de forma inadecuada. Él mismo, en un trabajo presentado en la ciudad de Mérida (Venezuela) y titulado: ¿De qué manera la FCM puede ser un factor para planear el entrenamiento? (Bouzas, 2008), sostiene:

Parece claro que la FCM es específica para cada tipo de ejercicio. Debido a esto, para una perfecta prescripción de ejercicio en la que se empleen ecuaciones de cálculo de la FCM, es necesario seleccionar aquellas ecuaciones que puedan estimar la FCM con mayor exactitud, ya que las ecuaciones que presentan un menor grado de precisión pueden inducir al entrenador a cometer graves errores metodológicos en la planificación del entrenamiento. (p. 97)

En este orden de ideas, es importante hacer notar que existen muchas ecuaciones predictivas para el cálculo de la FCME. En segundo término, se cuenta con ecuaciones predictivas para el cálculo de la FCME adaptables a diferentes disciplinas deportivas, y aún hay ecuaciones para que, en una misma disciplina deportiva, se trabaje con varias de ellas en tanto las especificidades y las demandas de cada disciplina y de cada deportista han de ser diferentes. Entre algunas de las fórmulas que recomiendan Bouzas y Delgado (2007) están:

\begin{tabular}{|l|l|}
\hline \multicolumn{1}{|c|}{ FÓRMULA } & \multicolumn{1}{c|}{ POBLACIÓN } \\
\hline FCME $=211-0,922($ EDAD $)$ & Hombres asintomáticos \\
\hline FCME $=200-0,71($ EDAD) & Hipertensos (as) \\
\hline FCME $=189-0,56($ EDAD) & Retardo mental \\
\hline FCME $=209-($ EDAD $)$ & Enfermedad oronaria \\
\hline FCME $=198-0,41($ EDAD) & Sedentarios (as) \\
\hline FCME $=217-0,85($ EDAD $)$ & Hombres y mujeres de peso normal \\
\hline FCME $=206,3-0,711(E D A D)$ & Deportistas de nivel nacional \\
\hline
\end{tabular}

Es de notar que aún existen muchas otras fórmulas propuestas para otro tipo de poblaciones e incluso con otras características, de acuerdo con los niveles de exigencia, bien sea de la actividad o la disciplina deportiva, si del caso se trata.

Finalmente se recomienda a profesores, estudiantes e instituciones de formación docente en el campo de la Educación Física, el Deporte, la Recreación y las Ciencias Aplicadas al Deporte, a entrenadores deportivos, a atletas, a monitores e instructores de actividad y ejercicio físico, aerobics, fitness, pilates, taebox, acuabox, e incluso a personas que se inician en la práctica regular de cualquier tipo de actividad física, entre otros, a trabajar sobre la base de nuevas investigaciones que en el campo de la fisiología del ejercicio muestran los avances que para esta materia se han alcanzado en los últimos años.

La revisión constante de estudios especializados y actualizados y de revistas científicas en el campo de la fisiología del ejercicio y otras ciencias aplicadas al deporte, ha de convertirse en una práctica habitual para todo el interesado en la delicada práctica de prescripción de ejercicio físico, ya sea en poblaciones susceptibles a niveles de alto rendimiento deportivo, como en aquellas otras poblaciones que realizan actividad física y la han adoptado como estilo de vida sin ningún otro interés. En tal sentido y en el caso específico de este trabajo, se recomienda la lectura de los (otros) trabajos que han sido citados en este texto y que podrían resultar de gran ayuda. 


\section{Referencias bibliográficas}

Abellán, J., Sainz, P. y Ortín, E. (2010). Guía para la prescripción de ejercicio físico en pacientes con riesgo cardiovascular. España: SEH-LELHA. Recuperado de: http://www.actasanitaria. com/fileset/doc 54883 FICHERO NOTICIA 9631.pdf

American Heart Association. (s. f.). Cardiovascular Diseases Statistics. [Estadísticas de Enfermedades Cardiovasculares]. Recuperado de: http://www.americanheart.org/presenter. jhtml?identifier $=4478$

Aracil, A. y Moyá, M. (2010, abril). Martii J. Karvonen: Enseñanzas y retos. RICYDE Revista Internacional en Ciencias del Deporte, 6(19), 81-82.

Bouzas, J., Delgado, M. (2004, mayo-junio). Comparação da freqüência cardíaca maxima por meio de provas com perfil aeróbico e anaeróbico. [Comparación de la frecuencia cardíaca máxima por medio de pruebas con perfil aeróbico y anaeróbico], Fitness \& Performance Journal, 3(3), 166-174. doi: 10.3900/fpj.3.3.166.p

Bouzas, J., Delgado, M. (2007). Empleo de ecuaciones para predecir la frecuencia cardiaca máxima en carrera para jóvenes deportistas. Archivos de Medicina del Deporte, 24(118), 112-120. Recuperado de http://femede.es/documentos/Original_Ecuaciones_112_118.pdf

Bouzas, J., Ottoline, N. y Delgado, M. (2010, octubre-diciembre). Aplicaciones de la frecuencia cardíaca máxima en la evaluación y prescripción de ejercicio físico. Apunts Medicina de L' Esport, 45(168), 251-258. doi 10.1016/j.apunts.2010.04.003

Bouzas, J. (junio, 2008). ¿De qué manera la FCM puede ser un factor para planear el entrenamiento? En J. G. Ramírez (Presidencia). VII Congreso Iberoamericano de Medicina del Deporte y Ciencias Aplicada. Congreso llevado a cabo en Mérida, Venezuela.

Freitas, G., Xavier, W., Silva-Junior, A. y Bouzas, J. (2003). Comparação da frequiência cardíaca máxima (FCM) calculada por 21 equações e FCM obtida em exercício de corrida em homens e mulheres [Comparación de la frecuencia cardiaca máxima (FCM), calculada por 21ecuaciones de la FCM obtenida en el ejercicio de carrera en hombres y mujeres]. Revista Mineira de Educação Fisica, 11(1), 149-158.

Garatachea, N., García, D. y de Paz, J. A. (2005, noviembre). Diferentes modelos de regresión para describir la relación $\mathrm{VO}_{2}-\mathrm{FC}$ y para estimar el $\mathrm{VO}_{2}$ a diferentes intensidades de esfuerzo. Revista de Ciencias de la Actividad Física y del Deporte, 1(3), Año 2.

Lagardera, F. y Lavega, P. (2003). Introducción a la praxiología motriz. Barcelona: Editorial Paidotribo, S. L.

Mujika, I. (2006). Métodos de cuantificación de las cargas de entrenamiento y competición. Kronos: Revista universitaria de la actividad física y el deporte, 5(10), 1-10. Recuperado de http:// www.revistakronos.com/docs/File/kronos/10/kronos_10 5.pdf 
Nogueira, S., Xavier, W., Figuereido, P., Bouzas, J. (2003). Comparação da freqüência cardíaca máxima (FCM) calculada por 21 equações e FCM obtida em natação estilo livre [Comparación de la frecuencia cardíaca máxima (FCM) calculada por 21 ecuaciones y la FCM obtenida en natación, estilo libre]. Revista Mineira de Educação Física, 11(1), 139-148.

Organización Mundial de la Salud [OMS]. (2008). Males cardiovasculares, primera causa de mиеrte. Recuperado de http://impreso.milenio.com/node/8122662

Organización Panamericana de la Salud [OPS]. (2007). Enfermedades cardiovasculares. Recuperado de http://new.paho.org/chi/index.php?option=com content\&task=view\&id=172\&Itemid=259

Popper, K. (1985). La lógica de la investigación científica. Editorial Tecnos: España.

Silva-Junior, A. y Bouzas, J. (2003). Comparação da freqüência cardíaca máxima obtida com a freqüência cardíaca máxima calculada por diversas fórmulas em exercício de cicloergómetro [Comparación de la frecuencia cardíaca máxima obtenida con la frecuencia cardíaca máxima calculada por diversas fórmulas en ejercicios de cicloergómetro]. Revista Mineira de Educacao Física, 11(1), 159-167.

Subiela, J. (2005). Introducción a la fisiología humana. Énfasis en la fisiología del ejercicio. Barquisimeto, Venezuela: FUNDAUPEL-IPB.

Valenzuela, R. (2007). Cuánto corre un jugador de fútbol en un partido. Recuperado de http:/l www.rubensvalenzuela.com/verArticulo.php?artId=3 